\title{
Acute effects of endurance exercise on jumping and kicking performance in top-class young soccer players
}

\author{
DANIEL JUÁREZ, CRISTINA LÓPEZ DE SUBIJANA, JAVIER MALLO, \& \\ ENRIQUE NAVARRO
}

Sport Biomechanics Laboratory, Faculty of Physical Activity and Sports Sciences, Technical University, Madrid, Spain

\begin{abstract}
The aim of this study was to examine the acute effects of endurance exercise on jumping and kicking performance in young soccer players. Twenty-one top-class young soccer players $(16.1 \pm 0.2$ years $)$ performed a countermovement jump test and a maximal instep soccer kick test before and after running for 20 min on a treadmill at $80 \%$ of their individual maximum heart rate. Two force platforms were used to obtain the following parameters during the countermovement jump: jump height, maximum power, maximum power relative to body mass, maximum vertical ground reaction force, maximum vertical ground reaction force relative to body mass, and maximum vertical ground reaction force applied to each leg. Maximum vertical ground reaction force and maximum vertical ground reaction force relative to body mass applied to the support leg during the kicks were also calculated with a force platform. The kicking motion was recorded using a three-dimensional motion-capture system. Maximum velocity of the ball, maximum linear velocity of the toe, ankle, knee and hip, and linear velocity of the toe at ball contact during the kicks were calculated. Non-significant differences were found in the parameters measured during the countermovement jump and the maximal instep soccer kick test before and after running, suggesting that the jumping and kicking performances of top-class young soccer players were not significantly affected after 20 min treadmill running at $80 \%$ of their individual maximum heart rate.
\end{abstract}

Keywords: fump, kick, soccer, fatigue

\section{Introduction}

In soccer, strength, power, and endurance are important for performance (Svensson \& Drust, 2005; Wisloff, Castagna, Helgerud, Jones, \& Hoff, 2004) and must be taken into account in training. However, the development of these physical capacities at the same time can be complicated. According to some authors (Bell, Syrotuik, Socha, Maclean, \& Quinney, 2000; Hennessy \& Watson, 1994; Kraemer et al., 1995), during the first weeks of training these physical capacities can be improved, but after $6-8$ weeks strength and power impairments are observed. Different researchers have examined the acute effects of endurance exercises on strength and power activities. In this regard, it has been observed that a previous long-term endurance exercise can adversely influence both strength and power (Chambers, Noakes, Lambert, \& Lambert, 1998; Lepers, Pousson, Maffiuletti, Martin, \& Van Hoecke, 2000; Millet et al., 2002; Nicol, Komi, \& Marconnet, 1991). Other researchers have reported a decrease in jumping and kicking performance under fatiguing conditions (Abt, Reaburn, Holmes, \& Gear, 2003; Hoffman, Nusse, \& Kang, 2003; Kellis, Katis, \& Vrabas, 2006; Lees \& Davies, 1988; Oliver, Armstrong, \& Williams, 2006; Perroni et al., 2006). In these studies, jumping or kicking performance was measured after a soccer match, an exercise protocol that simulated a soccer match or a muscular endurance exercise. However, to our knowledge, there is no evidence about the acute effects of moderate-volume endurance exercises on kicking and jumping performance in soccer players.

Correspondence: D. Juárez, Sport Biomechanics Laboratory, Faculty of Physical Activity and Sports Sciences, Technical University of Madrid, Avda. Martín Fierro 7, 28040 Madrid, Spain. E-mail: djuarezsg@yahoo.es 
The effects of moderate-volume endurance exercises on power do not seem to be clear in the literature. While some studies have reported non-significant changes in countermovement jump performance (Vuorimaa, Vasankari, \& Rusko, 2000), others have reported significant improvements (Boullosa \& Tuimil, 2007; Vuorimaa, Virlander, Kurkilahti, Vasankari, \& Häkkinen, 2006). Thus, it is reasonable to hypothesize that power is not reduced after moderate endurance training.

Since both power and endurance are important parameters in soccer, the effects of moderate-volume endurance exercises on power activities have to be taken into consideration, especially when training is periodized. The selection of the exercises, training loads, and their sequence should be appropriately organized to optimize performance. In a study of junior male elite soccer players (Helgerud, Engen, Wisloff, \& Hoff, 2001), an improvement of aerobic endurance was observed without significant differences in maximal vertical jump height, kicking velocity, strength, speed, kicking precision or quality of passes after a specific programme of training. In addition to habitual soccer training, this programme consisted in running $4 \times 4 \mathrm{~min}$ at $90-95 \%$ of maximum heart rate, with 3 min jogging between interval runs, twice a week for 8 weeks. However, the strength and power training loads and the training sequence of each session are not made clear by the authors. Good planning of training loads in each session should contribute to the improvement of these important physical capacities in soccer. Thus the aim of the present study was to examine the acute effects of a moderate endurance exercise on jumping and kicking performances in top-class young soccer players.

\section{Materials and methods}

\section{Participants}

Twenty-one top-class young soccer players (age $16.1 \pm 0.2$ years; height $1.77 \pm 0.06 \mathrm{~m}$; body mass $67.7 \pm 6.3 \mathrm{~kg}$ ) volunteered to participate in the study. All participants reported they were free from physical or orthopaedic injury, which would have prevented them from exerting maximal effort in a soccer kick. These soccer players belonged to a junior team of a Spanish First Division Club. Some of the players had played for the national team in junior categories. During the study, the participants trained 4 days a week and played a soccer match every weekend. The parents of the participants provided written informed consent allowing the players to take part in the study. The University Ethics Committee approved the protocol.

\section{Procedures}

All procedures were performed in the Sport Biomechanics Laboratory of the Faculty of Physical Activity and Sports Science of the Polytechnic University of Madrid (Spain). Reflective markers $(14 \mathrm{~mm}$ in diameter) were attached to 31 body landmarks as follows: four on the head, and one each on the spinous process of the 7 th cervical vertebrae where the clavicles meet the sternum, xiphoid process of the sternum, acromio-clavicular joints, lateral epicondyle approximating the joint axis of the elbows, wrists bar thumb side, wrists bar 5th finger side, anterior superior iliac spines, posterior superior iliac spines, lower lateral $1 / 3$ surface of the thighs, lower $1 / 3$ surface of the shanks to determine the alignment of the ankle flexion axis, lateral epicondyle of the knees, lateral malleolus along an imaginary line that passes through the transmalleolar axis, calcaneous, and toe placed over the fourth metatarsal head. These last two reflective markers were placed over the sport shoes. In addition, two markers were placed on the surface of the ball at diametrically opposite ends.

Once the markers were in position, the participants performed a 15-min standardized warm-up and then two pre-tests: a countermovement jump and a maximal instep soccer kick. The warm-up consisted of 5-min jogging followed by some stretching exercises for the psoas, quadriceps, hip adductors, and calf muscles $(2 \times 8 \mathrm{~s}$ for each leg). Then the participants performed eight submaximal countermovement jumps and eight submaximal kicks.

The countermovement jump is a maximal vertical jump from an erect standing position, with the legs separated shoulder-width and the hands placed on the waist. After receiving a verbal instruction ("ready"), the participants carried out a maximal vertical jump without taking the hands off the waist. The participants placed one leg on each force platform, to obtain the maximum vertical ground reaction force applied for each leg. Three countermovement jumps were performed with $15 \mathrm{~s}$ recovery between jumps. The reaction force applied on the ground during the jump was registered using two synchronized extensometric force platforms (Dinascan, IBV, Valencia, Spain) operating at 250 Hz. A low-pass digital filter was used to eliminate noise due to the electronic operation of the force platforms. Raw data were processed using a $50-\mathrm{Hz}$ low-pass filter. Jump height was calculated from the impulse and the trial with the greatest height jumped was selected for further analysis.

In the maximal instep soccer kick test, the participants performed a 4- to 5-m approach run, kicking the ball with the dominant leg towards a net located $5 \mathrm{~m}$ in front of them (Markovic, Dizdar, \& 
Jaric, 2006). The ball was placed such that both a right- and left-footed player could impact with his support leg on the force platform. Three trials were performed with a 30-s interval between trials (Markovic et al., 2006). A FIFA (Fédération Internationale de Football Association) standard ball was used for all the kicks ensuring that the pressure of inflation remained the same throughout the trials.

The kicking test was recorded using a threedimensional motion-capture system (VICON Motion Systems, Oxford Metrics Ltd., Oxford, UK) with six cameras operating at $250 \mathrm{~Hz}$ (Figure 1). Calibration residuals were determined in accordance with VICON's guidelines with a position data accuracy of $1 \mathrm{~mm}$. Raw data were filtered using Quintic spline functions with the Woltring-CGV method for calculating the adjustment factor. This kicking set-up facilitated the registration of threedimensional coordinates of the markers and the reaction forces of the player without affecting the performance (i.e. with high external validity). The kicks for which the ball was released with the highest speed were selected for further analysis.

After the pre-test, participants ran on a treadmill for $20 \mathrm{~min}$ at $80 \%$ of their individual maximum heart rate, calculated previously during an incremental running test carried out in their home club. This heart rate corresponds to the aerobic-anaerobic transition zone, approximately $90-95 \%$ of the anaerobic threshold for trained individuals (Kindermann, Simon, \& Keul, 1979). Heart rate monitors (Polar RS800, Polar Electro, Finland) were used to calculate the exercise intensity, adapting the treadmill speed individually to allow the participants to remain close to the target exercise intensity. This exercise intensity was chosen based on the study of Gorostiaga et al. (2004), in which young soccer players undertook endurance training once a week for 11 weeks with $25-\mathrm{min}$ runs at a velocity close to the individual anaerobic threshold. It has also been found in professional soccer players that submaximal exercise running bouts can be a valid soccer-specific test to measure endurance capacity, as individual values of blood lactate concentration during submaximal running were correlated with the individual values of distance covered during competitive matches (Bangsbo \& Lindquist, 1992).

After the endurance exercise, the participants repeated (post-test) the countermovement jump test and the maximal instep soccer kick test. Jump height, absolute and relative to body mass maximum power, and the vertical ground reaction force (absolute, relative, and applied to each supporting leg) were calculated during the countermovement jump. In addition, during the kicking test the maximum velocity of the ball, toe, ankle, knee, and hip were calculated. The linear velocity of the toe at ball contact was also calculated. The absolute and relative maximum vertical ground reaction force applied to the supporting leg complemented the analysis.

\section{Statistical analyses}

The SPSS v. 14.0 software package (SPSS Inc., Chicago, IL) was used for the analysis of the data. Normality of the sample was tested by means of the Kolmogorov-Smirnov test. Means and standard deviations are reported. The intra-class correlation coefficient between trials and the coefficient of variation (standard deviation $/$ mean) ${ }^{\star} 100$ were also determined. Student's $t$-tests with Bonferroni correction were used to analyse differences between the pre- and post-tests. Statistical significance was set at $P<0.003$.

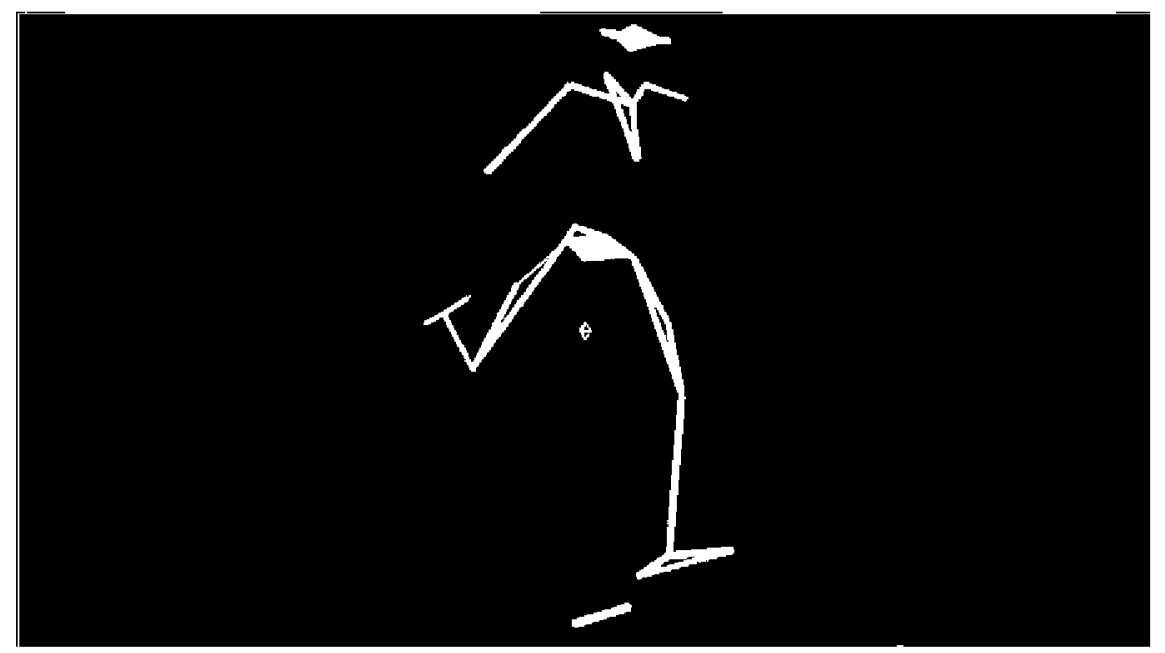

Figure 1. Soccer kick recorded and analysed with the three-dimensional motion-capture system (VICON Motion Systems, Oxford Metrics Ltd., Oxford, UK). 


\section{Results}

All the parameters measured in this study showed an intra-class correlation coefficient between trials $>0.8$ and a coefficient of variation $<6 \%$.

Non-significant differences were observed between countermovement jumps performed before and after running for $20 \mathrm{~min}$. A jump height of 0.41 $\mathrm{m}(s=0.08)$ was obtained in the pre-test and a jump height of $0.43 \mathrm{~m}(s=0.09)$ in the post-test. The maximum power registered in the countermovement jump was $3658 \mathrm{~W}(s=557)$ in the pre-test and 3938 $\mathrm{W}(s=704)$ in the post-test. The maximum power relative to body mass was $54.67 \mathrm{~W} \cdot \mathrm{kg}^{-1}(s=6.69)$ in the pre-test and $58.09 \mathrm{~W} \cdot \mathrm{kg}^{-1}(s=8.02)$ in the post-test. A maximum vertical ground reaction force of $1670 \mathrm{~N}(s=167)$ was recorded in the pre-test and a maximum vertical ground reaction force of $1671 \mathrm{~N}$ $(s=208)$ in the post-test. The maximum vertical ground reaction force relative to body mass was $2.54^{\star} \mathrm{BM}$ (Body Mass) $(s=0.27)$ in the pre-test and $2.54^{\star} \mathrm{BM}(s=0.25)$ in the post-test. Analysis of the maximum vertical ground reaction force applied to the support leg showed for the dominant leg kick a value of $860 \mathrm{~N}(s=99.0)$ and $856 \mathrm{~N}(s=126)$ in the pre-test and post-test, respectively. For the other leg, the values were $810 \mathrm{~N}(s=92.9)$ in the pre-test and $815 \mathrm{~N}(s=105)$ in the post-test.

For the kick parameters, non-significant differences were also found between pre- and post-tests (Table I).

\section{Discussion}

The results of this study suggest that, among topclass young soccer players, countermovement jump and maximal instep soccer kick performances were not significantly altered after a $20-\mathrm{min}$ run at $80 \%$ of individual maximum heart rate. In the present study, we did not detect significant differences in countermovement jump height before and after exercise, reinforcing previous observations that countermovement jump height was not affected by endurance activity. Vuorimaa et al. (2000) reported no significant changes in the jump height of ten national-level middle-distance runners after two intermittent exercise protocols at the velocity associated with $\dot{V} \mathrm{O}_{2 \text { max }}$ : fourteen 60 -s runs with $60 \mathrm{~s}$ rest between runs and seven 120 -s runs with 120 s rest between runs. It has also been reported that countermovement jump performance of middle-distance runners does not decrease significantly until a blood lactate concentration of $\sim 10 \mathrm{mmol} \cdot 1^{-1}$ (Rusko, Nummela, \& Mero, 1993). In contrast, we observed a significant improvement in the absolute and relative maximum power during the countermovement jump. These data show that the parameters measured in a countermovement jump must be analysed independently when coaches assess explosive strength.

Several authors have reported negative effects of aerobic exercise on strength capacity. Lepers et al. (2000) reported a $10 \%$ decrease $(P<0.01)$ in countermovement jump height in male triathletes after $2 \mathrm{~h}$ of running at $75 \%$ of individual maximum aerobic velocity. In addition, a decrease in drop jump capacity was reported in experienced endurance athletes after performing a marathon (Nicol et al., 1991). Moreover, submaximal aerobic activity has been shown to affect other explosive strength parameters, such as squat jump height. In a study of eight male athletes (age $46 \pm 7$ years), Chambers et al. (1998) registered a decrease of power strength after $90 \mathrm{~km}$ of running. However, the above studies showed a significant effect of aerobic exercise on power performance because they used much longer endurance protocols compared with the present

Table I. Kick parameters (mean $\pm s$ )

\begin{tabular}{llr}
\hline Parameter & Test & Value \\
\hline Maximum velocity of the ball $\left(\mathrm{m} \cdot \mathrm{s}^{-1}\right)$ & Pre-test & $30.06 \pm 1.54$ \\
& Post-test & $30.33 \pm 1.44$ \\
Maximum linear velocity of the toe $\left(\mathrm{m} \cdot \mathrm{s}^{-1}\right)$ & Pre-test & $24.59 \pm 1.33$ \\
& Post-test & $24.43 \pm 1.60$ \\
Maximum linear velocity of the ankle $\left(\mathrm{m} \cdot \mathrm{s}^{-1}\right)$ & Pre-test & $19.23 \pm 0.88$ \\
& Post-test & $19.30 \pm 1.02$ \\
Maximum linear velocity of the knee $\left(\mathrm{m} \cdot \mathrm{s}^{-1}\right)$ & Pre-test & $11.04 \pm 0.58$ \\
& Post-test & $11.12 \pm 0.54$ \\
Maximum linear velocity of the hip $\left(\mathrm{m} \cdot \mathrm{s}^{-1}\right)$ & Pre-test & $5.48 \pm 0.58$ \\
Linear velocity of the toe at ball contact $\left(\mathrm{m} \cdot \mathrm{s}^{-1}\right)$ & Post-test & $5.43 \pm 0.45$ \\
& Pre-test & $18.63 \pm 3.27$ \\
Maximum vertical ground reaction force applied to support leg $(\mathrm{N})$ & Post-test & $18.53 \pm 2.76$ \\
& Pre-test & Post-test \\
Maximum vertical ground reaction force relative to body mass & Pre-test & $2496.29 \pm 690.51$ \\
& Post-test & $2538.08 \pm 580.63$ \\
\end{tabular}

Note: $\mathrm{BM}=$ Body Mass. 
study, and they assessed triathletes and distance runners who may not be as explosive as the present soccer players.

In the present study, the speed at which the ball was released after a maximal kick was $\sim 30 \mathrm{~m} \cdot \mathrm{s}^{-1}$. This value is slightly higher than that reported in other studies of young soccer players (Mognoni, Narici, Sirtori, \& Lorenzelli, 1994). It should be noted that in the present study, the players kicked the ball without the need for accuracy, whereas other kick tests demand both accuracy and maximum speed of the ball.

The comparison of ball speed before and after the experimental treatment did not reveal any significant differences. Therefore, we can state that $20 \mathrm{~min}$ of moderate-intensity exercise does not affect the maximum kicking speed of young soccer players. In a study of ten male amateur soccer players, Kellis et al. (2006) reported that ball velocity decreased significantly $(P<0.01)$ after a $90-$ min intermittent exercise protocol. A significant $(P<0.05)$ reduction in ball velocity was also observed in seven male adult soccer players after repeated loaded knee extension $(40 \%$ body mass) and flexion (50\% body mass) motions on a weight-training machine until exhaustion (Apriantono, Nunome, Ikegami, \& Sano, 2006). The ball velocity attained by five skilled male soccer players was significantly greater before performing a 6-min step test from a height of $0.5 \mathrm{~m}$ at a cadence of 30 steps per minute, than after the step test (Lees \& Davies, 1988).

When considering the maximum linear velocity of the joint markers in the kicking leg (hip, knee, ankle and toe) and the linear velocity of toe at ball contact, non-significant differences were observed between pre- and post-tests. In another study, eight professional soccer players completed $6 \times 15$-min activity bouts with a 15 -min passive half-time interval. At rest and subsequently at the 15 -min intervals, the players completed a maximal velocity kick test. Although a fatigue effect was observed, due to a kinematic alteration in technique, foot velocity at ball contact was maintained during the protocol (Greig, Lovell, \& Siegler, 2007).

The maximum vertical ground reaction force applied to the support leg during the kick was $2500 \mathrm{~N}$. When this parameter was related to individual body mass, we observed that the participants manifested forces approximately 3.7 times their body mass, without differences between tests.

In soccer, the effect of endurance exercise depends on the volume and intensity of the load imposed on the players. It would appear that moderate-intensity and moderate-volume endurance exercise does not adversely affect power in the same way as high-volume aerobic exercise. This kind of exercise is typical of pre-season training, when players experience the overall highest training volume. The results presented here might have important implications for soccer coaches, helping them to organize the sequence in which the different drills are performed during a training session, in order to optimize the periodization and to develop methods to avoid the possible adverse effects of training.

Due to the peculiarities of competitive exercise, it would be interesting to study the effects of intermittent exercise, which is much more specific to the demands of the game, on the technical and power capacities of soccer players. Thus, further research is required to determine the influence of different kinds of endurance exercise on the power development of soccer players, as it is a key component of physical performance.

\section{Conclusions}

In top-class young soccer players, jumping and kicking performances were not significantly affected after running for $20 \mathrm{~min}$ on a treadmill at $80 \%$ of individual maximum heart rate. The results of the present study indicate that certain endurance exercises can be carried out before performing some explosive strength exercises without interfering with them.

\section{References}

Abt, G., Reaburn, P., Holmes, M., \& Gear, T. (2003). Changes in peak sprint speed during prolonged high-intensity intermittent exercise that simulates team sport play. Fournal of Sports Sciences, 21, 256-257.

Apriantono, T., Nunome, H., Ikegami, Y., \& Sano, S. (2006). The effect of muscle fatigue on instep kicking kinetics and kinematics in association football. Fournal of Sports Sciences, $24,951-960$.

Bangsbo, J., \& Lindquist, F. (1992). Comparison of various exercise tests with endurance performance during soccer in professional players. International fournal of Sports Medicine, 13, $125-132$.

Bell, G., Syrotuik, D., Socha, T., Maclean, I., \& Quinney, H. A. (2000). Effect of strength training and concurrent strength and endurance training on strength, testosterone and cortisol. fournal of Strength and Conditioning Research, 11, 57-64.

Boullosa, D. A., \& Tuimil, J. L. (2007). Acute effect of two different running protocols on jump capacity in distance runners. Paper presented at the 12th Annual Congress of the European College of Sport Science, Jyväskylä, 11-14 July.

Chambers, C., Noakes, T. D., Lambert, E. V., \& Lambert, M. I. (1998). Time course of recovery of vertical jump height and heart rate versus running speed after a $90-\mathrm{km}$ foot race. Fournal of Sports Sciences, 16, 645-651.

Gorostiaga, E., Izquierdo, M., Ruesta, M., Iribarren, J., González-Badillo, J. J., \& Ibáñez, J. (2004). Strength training effects on physical performance and serum hormones in young soccer players. European fournal of Applied Physiology, 91, 698-707.

Greig, M., Lovell, R., \& Siegler, J. (2007). Influence of soccerspecific fatigue on kinematics of kicking. Fournal of Sports Science and Medicine, 10 (suppl.), 149-150. 
Helgerud, J., Engen, L. C., Wisloff, U., \& Hoff, J. (2001). Aerobic endurance training improves soccer performance. Medicine and Science in Sports and Exercise, 33, 1925-1931.

Hennessy, L. C., \& Watson, A. W. S. (1994). The interference effects of training for strength and endurance simultaneously. Fournal of Strength and Conditioning Research, 8, 12-19.

Hoffman, J., Nusse, V., \& Kang, J. (2003). The effect of an intercollegiate soccer game on maximal power performance. Canadian fournal of Applied Physiology, 28, 807-817.

Kellis, E., Katis, A., \& Vrabas, I. S. (2006). Effects of an intermittent exercise fatigue protocol on biomechanics of soccer kick performance. Scandinavian fournal of Medicine and Science in Sports, 16, 334-344.

Kindermann, W., Simon, G., \& Keul, J. (1979). The significance of the aerobic-anaerobic transition for the determination of work load intensities during endurance training. European fournal of Applied Physiology, 42, 25-34.

Kraemer, W. J., Patton, J. F., Gordon, S. E., Harman, E. A., Deschenes, M. R., Reynolds, K., et al. (1995). Compatibility of high-intensity strength and endurance training on hormonal and skeletal muscle adaptations. Fournal of Applied Physiology, 78, 976-989.

Lees, A., \& Davies, T. (1988). The effects of fatigue on soccer kick kinematics. Fournal of Sports Sciences, 6, 156-157.

Lepers, R., Pousson, M. L., Maffiuletti, N. A., Martin, A., \& Van Hoecke, J. (2000). The effects of a prolonged running exercise on strength characteristics. International fournal of Sports Medicine, 21, 275-280.

Markovic, G., Dizdar, D., \& Jaric, S. (2006). Evaluation of tests of maximum kicking performance. Fournal of Sports Medicine and Physical Fitness, 46, 215-220.

Millet, G. Y., Lepers, R., Maffiuletti, N. A., Babault, N., Martin, V., \& Lattier, G. (2002). Alteration of neuromuscular function after an ultramarathon. Fournal of Applied Physiology, 92, 486-492.

Mognoni, P., Narici, M. V., Sirtori, M. D., \& Lorenzelli, F. (1994). Isokinetic torques and kicking maximal ball velocity in young soccer players. Fournal of Sports Medicine and Physical Fitness, 34, 357-361.

Nicol, C., Komi, P., \& Marconnet, P. (1991). Fatigue effects of marathon running on neuromuscular performance - I. Changes in muscle force and stiffness characteristics. Scandinavian Fournal of Medicine and Science in Sports, 1, $10-17$.

Oliver, J. L., Armstrong, N., \& Williams, C. A. (2006). Changes in jump performance and muscle activity following prolonged footballspecific exercise. Paper presented at the 11 th Annual Congress of the ECSS, Lausanne, 5-8 July.

Perroni, F., Tessitore, A., Pesce, C., Cortis, C., Lupo, C., \& Capranica, L. (2006). Interlimb coordination, strength, and power performance following a soccer match. Paper presented at the 11th Annual Congress of the ECSS, Lausanne, 5-8 July.

Rusko, H., Nummela, A., \& Mero, A. (1993). A new method for the evaluation of anaerobic running power in athletes. European fournal of Applied Physiology and Occupational Physiology, 66, 97-101.

Svensson, M., \& Drust, B. (2005). Testing soccer players. Fournal of Sports Sciences, 23, 601-618.

Vuorimaa, T., Vasankari, T., \& Rusko, H. (2000). Comparison of physiological strain and muscular performance of athletes during two intermittent running exercises at the velocity associated with $\dot{V} \mathrm{O}_{2 \mathrm{max}}$. International fournal of Sports Medicine, 21, 96-101.

Vuorimaa, T., Virlander, R., Kurkilahti, P., Vasankari, T., \& Häkkinen, K. (2006). Acute changes in muscle activation and leg extension performance after different running exercises in elite long distance runners. European fournal of Applied Physiology, 96, 282-291.

Wisloff, U., Castagna, C., Helgerud, J., Jones, R., \& Hoff, J. (2004). Strong correlation of maximal squat strength with sprint performance and vertical jump height in elite soccer players. British Fournal of Sport Medicine, 38, 285-288. 